\title{
Anti-metabolic syndrome effects of adenosine ingestion in stroke-prone spontaneously hypertensive rats fed a high-fat diet
}

\author{
Ardiansyah $^{1}{ }^{*}$, Hitoshi Shirakawa ${ }^{1}$, Yumi Sugita ${ }^{1}$, Takuya Koseki ${ }^{2}$ and Michio Komai ${ }^{1}$ \\ ${ }^{1}$ Laboratory of Nutrition, Graduate School of Agricultural Science, Tohoku University, 1-1 Tsutsumidori-Amamiyamachi, \\ Aoba-ku, Sendai 981-8555, Japan \\ ${ }^{2}$ Department of Bioresource Engineering, Faculty of Agriculture, Yamagata University, Japan
}

(Received 11 September 2009 - Revised 14 December 2009 - Accepted 14 January 2010 - First published online 23 February 2010 )

We have demonstrated previously that both acute and chronic oral administration of adenosine have novel functions such as anti-hypertensive effects and improved hyperlipidaemia in stroke-prone spontaneously hypertensive rats (SHRSP) fed a normal diet. The purpose of the present study was to investigate the effect of adenosine administration on metabolic syndrome-related parameters in SHRSP fed a high-fat diet. Six-week-old rats were divided into three groups, and were administered either water (control) or adenosine (10 or $100 \mathrm{mg} / \mathrm{l})$ for 8 weeks. During this period, the rats had free access to a high-fat diet based on AIN-93M. The results showed that hypertension, plasma lipid, NO, insulin, glucose and urinary 8-hydroxy-2'-deoxyguanosine levels improved significantly in both adenosine groups. The mRNA expression levels of genes involved in anti-oxidative activity and adenosine receptors were also altered in the adenosine groups. Administration of adenosine also increased plasma adiponectin levels, accompanied by upregulation of mRNA expression level of adiponectin and adiponectin receptor 1 in perirenal fat and adiponectin receptor 2 in the liver. In conclusion, oral administration of adenosine is effective for improving metabolic syndrome-related parameters in SHRSP, and accordingly it may prevent the progression of the metabolic syndrome.

High-fat diet: Blood pressure: Glucose metabolism: Lipid profile

Obesity has become increasingly prevalent worldwide as a result of changes in lifestyle, especially eating habits, and it is involved in the aetiology of a number of conditions such as CVD, hypertension, stroke and diabetes ${ }^{(1,2)}$. Overconsumption of high-energy food and lower energy expenditure have resulted in an alarming increase in the incidence of obesity ${ }^{(3)}$. The fact that clinically diagnosed insulin resistance, hypertension, increased fat distribution and high plasma TAG levels have been associated with the development of diseases associated with metabolic syndrome-related parameters has attracted attention from the scientific community. Prevention and improvement of obesity and metabolic syndrome-related diseases are therefore important issues in modern society, and represent an effective strategy for the promotion of better health.

Adenosine is an endogenous purine nucleoside that modulates many physiological processes, with these effects being mediated by the activation of specific subtypes of adenosine receptors, termed $\mathrm{A}_{1}, \mathrm{~A}_{2 \mathrm{~A}}, \mathrm{~A}_{2 \mathrm{~B}}$ and $\mathrm{A}_{3}^{(4,5)}$. Adenosine can be extracted from plant and mammalian tissues. Higher adenosine concentrations in the plant and mammalian tissues result from enhanced excretion of adenosine or diminished uptake of adenosine ${ }^{(6)}$.

Previous studies have provided evidence to support the physiological role of adenosine in general health. For example, studies in both rats and human subjects showed that oral administration of adenosine in sucrose solutions decreased blood glucose and insulin concentrations due to its inhibitory effect on $\alpha$-glucosidase ${ }^{(7,8)}$. Continuous intravenous infusion of adenosine in premature infants was also reported to have dramatic beneficial effects on neonatal refractory pulmonary hypertension $^{(9)}$, in addition to attenuating the proliferation of both human and rat glomerular mesangial cells associated with hypertension and diabetes ${ }^{(10)}$.

In a previous study in stroke-prone spontaneously hypertensive rats (SHRSP) fed a normal diet, we showed that acute and chronic oral administration of adenosine induced novel effects such as lowering of blood pressure (BP), improvement of hyperlipidaemia and increase in plasma adiponectin levels accompanied by alleviation of hyperinsulinaemia ${ }^{(11)}$. Recent studies in mice also demonstrated the role of adenosine and its receptors in the regulation of hepatic fibrosis $(12,13)$ and peripheral lipid metabolism ${ }^{(14)}$. However, the detailed mechanisms underlying the effects of adenosine on metabolic syndrome-related parameters in rats have not been clarified, especially its effect on adenosine and adiponectin receptors. The purpose of the present study was therefore to extend these previous investigations by determining the effect of adenosine administration on metabolic syndrome-related parameters in

Abbreviations: Ad10, adenosine $10 \mathrm{mg} / \mathrm{l}$; Ad100, adenosine $100 \mathrm{mg} / \mathrm{l}$; 8-OHdG, 8-hydroxy-2'-deoxyguanosine; BP, blood pressure; SHRSP, stroke-prone spontaneously hypertensive rats; TC, total cholesterol.

* Corresponding author: Ardiansyah, fax +81 22717 8813, email ardy@biochem.tohoku.ac.jp 
SHRSP fed a high-fat diet. SHRSP fed a high-fat diet serve as a suitable animal model of hypertension-related disorders that are similar to human essential hypertension, hyperlipidaemia and insulin resistance.

\section{Material and methods}

\section{Animal experiments and diet}

Male SHRSP/Izumo strain (Japan SLC, Shizuoka, Japan) were used in the present study. The rats were housed individually in stainless steel cages under a controlled atmosphere (temperature, $23 \pm 2{ }^{\circ} \mathrm{C}$; humidity, $50 \pm 10 \%$; $12 \mathrm{~h}$ light-dark cycle, 08.00-20.00 hours). After a 1-week acclimatisation period, the six-week-old SHRSP were divided into either a control group or two groups administered adenosine based on their body weight. All the rats were fed an AIN-93M-based diet supplemented with $20 \%$ lard as the source of increased fat (Table 1). The rats in the control group were provided with distilled water for 8 weeks, while the rats in the adenosine (Wako Pure Chemical Co., Osaka, Japan) groups were administered distilled water containing either $10 \mathrm{mg} / \mathrm{l}$ adenosine (Ad10) or $100 \mathrm{mg} / \mathrm{l}$ adenosine (Ad100) over the same period. Water and food intakes were recorded every day. The systolic BP and body weight were measured every week during the experimental period. At the end of the experimental period, the rats were fasted for $16 \mathrm{~h}$ and were then sacrificed under light diethyl ether anaesthesia. Blood samples were collected and centrifuged at $1870 \mathrm{~g}$ for $15 \mathrm{~min}$ at $4^{\circ} \mathrm{C}$ in a centrifuge (CF7D2; Hitachi Co. Ltd, Tokyo, Japan). The livers were excised promptly and washed with ice-cold isotonic saline. Both plasma and liver tissue samples were stored at $-80^{\circ} \mathrm{C}$ until required for later analyses.

\section{Ethical guidelines}

The experiments did not involve human subjects or patients. The experimental plan for the present study was approved by the Animal Research-Animal Care Committee of Tohoku University. The entire experiment was carried out in accordance with the guidelines issued by this committee and the Japanese government legislation (2005). The same committee supervised the care and use of the rats used in the present study.

Table 1. Composition of the experimental diets based on the AIN-93 diet

\begin{tabular}{lc}
\hline Ingredient $(\mathrm{g} / \mathrm{kg})^{\star}$ & Control \\
\hline tert-Butylhydroquinone & $0 \cdot 008$ \\
L-Cystine & $1 \cdot 8$ \\
Choline bitartrate & $2 \cdot 5$ \\
Vitamin mixture & 10 \\
Mineral mixture & 35 \\
Lard & 200 \\
Cellulose & 50 \\
Sucrose & 100 \\
Casein & 140 \\
Maize starch & $460 \cdot 692$
\end{tabular}

* tert-Butylhydroquinone, L-cystine, choline bitartrate and soyabean oil were purchased from Wako Pure Chemical Co, and vitamin and mineral mixtures, cellulose, sucrose and casein were obtained from the Oriental Yeast Co. (Tokyo, Japan).

\section{Blood pressure measurements}

BP was measured by the tail-cuff method using a BP meter without warming (MK-2000; Muromachi Kikai, Tokyo, Japan) as described previously ${ }^{(15)}$. A minimum of six BP measurements were obtained for each rat. The mean value of four consistent readings of systolic BP was regarded as the individual systolic BP.

\section{Plasma and liver parameters}

Plasma levels of blood urea nitrogen, creatinine, albumin, glucose, total cholesterol (TC), TAG, NEFA and HDL-cholesterol were determined by enzymatic colorimetric methods (Wako Pure Chemical Co.). Plasma adiponectin levels were measured using a rat adiponectin ELISA kit obtained from Otsuka (Otsuka Co., Tokyo, Japan), and plasma insulin levels were measured using a rat insulin ELISA kit purchased from Shibayagi (Shibayagi Co., Gunma, Japan). Plasma NO level was quantified by the Griess method $\left[\mathrm{NO}_{2} / \mathrm{NO}_{3}\right.$ Assay kit-C II (Colorimetric) Dojindo, Kumamoto, Japan] asdescribed previously $^{(15)}$. Urinary 8-hydroxy-2'-deoxyguanosine (8-OHdG) level was measured by an ELISA (New 8-OHdG Check; Institute for the Control of Aging, Shizuoka, Japan) as described previously ${ }^{(15)}$. The concentration of LDL-cholesterol was calculated by Friedewald's formula ${ }^{(16)}$. Liver total lipids were measured according to the Folch method ${ }^{(17)}$, and liver TC and TAG concentrations were determined using a kit that was the same as that used for determining plasma $\mathrm{TC}$ and $\mathrm{TAG}$ concentrations.

\section{Oral glucose tolerance test}

Oral glucose tolerance tests were conducted on 11-week-old SHRSP after fasting for $16 \mathrm{~h}$. Blood for glucose measurement was collected from the tail vein 30,60 and $120 \mathrm{~min}$ before and after SHRSP were $(1.8 \mathrm{~g} / \mathrm{kg}$ body weight) via a gastric tube. Plasma glucose and insulin levels were measured as described earlier. The analysis of the incremental area under the curve of the plasma glucose and insulin response was done on the basis of the method of Wolever \& Jenkins ${ }^{(18)}$.

\section{$R N A$ preparation and quantitative $R T-P C R$}

Total RNA was isolated from the liver and perirenal fat with a guanidine isothiocyanate-based reagent, Isogen (Nippon Gene Co., Tokyo, Japan), according to the instruction manual. Measurement of the wavelength ratio at 260 and $280 \mathrm{~nm}$ and agarose gel electrophoresis were performed for quantitative and qualitative analyses of the isolated RNA. Due to the low value of the ratio of perirenal fat mRNA at 260 and $280 \mathrm{~nm}$, we performed a RNA cleanup procedure based on RNeasy (Qiagen, Tokyo, Japan). Five micrograms of total RNA were used as a template to synthesise the cDNA. The RNA was denatured with oligo-dT/random primers, followed by incubation in $10 \mathrm{mmol} / \mathrm{l} \mathrm{dNTP}$ (Amersham Biosciences) and distilled water at $65^{\circ} \mathrm{C}$ for $5 \mathrm{~min}$. The RNA was then incubated in $50 \mathrm{mmol} / \mathrm{l}$ Tris- $\mathrm{HCl}$ buffer ( $\mathrm{pH} 8 \cdot 3$ ), $0 \cdot 1 \mathrm{M}$ DTT containing 50 units of SuperScript III reverse transcriptase (Invitrogen, Carlsbad, CA, USA) and 20 units of RNaseOUT RNase inhibitor (Invitrogen) in a total volume of $20 \mu \mathrm{l}$ at $25^{\circ} \mathrm{C}$ for 
Table 2. Sequences of the PCR primers for amplification

\begin{tabular}{lll}
\hline Gene & \multicolumn{1}{c}{ Forward primer } & \multicolumn{1}{c}{ Reverse primer } \\
\hline Ef-1 & GATGGCCCCAAATTCTTGAAG & GGACCATGTCAACAATTGCAG \\
Fasn & GGCTCACACACCTACGTATTGG & TGCTTAATGAAGAAGCATATGGCTT \\
Ppary & GGCCATATTATAGCTGTCATTATTCTC & GCAGCAGGTTGTCTTGGATGT \\
Srebp1c & GGAGCCATGGATTGCACATT & GCTTCCAGAGAGGAGCCAG \\
Sod & GGCCGTACTATGGTGGTCCA & TCCACCTTTGCCCAAGTCAT \\
Gshpx & TGACCGACCCCAAGTACATCA & AAATGTCGTTGCGGGACAC \\
Adipoq & TCCTGGTCACAATGGGATACC & TCCAGGAGTGCCATCTCTGC \\
Adipor1 & CCAAAAGTTCCAGGACTCAGGA & GAGGAACAGGAGCGCTTGC \\
Adipor2 & GTCTGGTTTTAGGCACTCCTTTG & CCCTGCCCCCATGTCTTAA \\
Adora2a & CAGGAAGGCCAAGAGCACC & CTCYAGCCTGGACCAGATGACC \\
Adora2b & CCAAGCATGTGGCCCACCT & AGACTTGTGTGACTCCTGGCAA \\
Adora3 & ACTTCTATGCCTGCCTTTTCATGT & CGTGGGTGAAGACCAGAAGC \\
\hline
\end{tabular}

Ef-1, eukaryotic elongation factor-1 $\alpha 1$; Fasn, fatty acid synthase; Ppar $\gamma$, PPAR $\gamma$; Srebp1c, sterol regulatory element-binding protein-1c, Sod, superoxide dismutase; Gshpx, glutathione peroxidase; Adipoq, Adiponectin; Adipor1, adiponectin receptor 1; Adipor2, adiponectin receptor 2; Adora2a, adenosine $A_{2 A}$ receptor; Adora2b, adenosine $A_{2 B}$ receptor; Adora3, adenosine $A_{3}$ receptor.

$5 \mathrm{~min}, 50^{\circ} \mathrm{C}$ for $60 \mathrm{~min}$ and $70^{\circ} \mathrm{C}$ for $15 \mathrm{~min}$. Aliquots of the cDNA were used as a template for the following quantitative PCR using an Applied Biosystems 7300 Real-Time PCR System (Foster City, CA, USA) according to the manufacturer's instructions. The genes listed in Table 2 were amplified by cDNA-specific primers using the SYBR Premix Ex Taq solution (Takara Bio Inc., Shiga, Japan). The relative levels of gene expression were normalised against the mRNA expression level of eukaryotic elongation factor- $1 \alpha 1^{(19)}$.

\section{Statistical analysis}

Values are presented as means with their standard errors. The differences between the groups studied were evaluated using one-way ANOVA followed by the Fisher post hoc test. Statistical Analysis Systems software (StatView-J 5.0; 1998 SAS Institute Inc., Cary, NC, USA) was used for all the statistical analyses. Probability values of $P<0.05$ were considered as statistically significant.

\section{Results}

\section{Body weight and daily intake}

The consumption of a high-fat diet for 8 weeks resulted in a further increase in body weight in SHRSP, with an approximate $20 \%$ increase compared with that observed in our previous studies on normal diets; the final body weight of SHRSP fed AIN-93M standard diet was 255.6 (SEM 3.7) $\mathrm{g}^{(15)}$. At the end of the experimental period, the final body weights were 297.0 (SEM 4.2), 290.3 (SEM 3.3) and 284.5 (SEM 8.6) g for the control, Ad10 and Ad100 groups, respectively. There was no difference in the gain in body weight, food or water intake and relative liver weight between the three groups (data not shown). We found that the relative perirenal fat weights for the control, Ad10 and Ad100 groups were 1.8 (SEM 0.1), 1.5 (SEM 0.1) and 1.7 (SEM 0.1) g/100 g body weight, respectively. A low dose of adenosine (Ad10) did not show a significant difference from the other two groups. The mean daily adenosine intake $(\mathrm{mg} / \mathrm{d}$ ) was 0.18 (SEM 0.02) in the Ad10 group and 2.1 (SEM 0.4) in the Ad100 group.

\section{Effects of adenosine on blood pressure and} plasma nitric oxide levels

The changes in the systolic BP of the rats during the experimental period are shown in Fig. 1(a). Compared with the control group, administration of adenosine at 10 and $100 \mathrm{mg} / \mathrm{l}$ was associated with significant decreases in BP starting from 7 weeks of age until the end of the experimental period. At the end of the experimental period, mean systolic BP was $209 \cdot 5$ (SEM 5.5), 191.5 (SEM 2.6) and 184.3 (SEM 4.2) $\mathrm{mmHg}$ for the control, Ad10 and Ad100 groups, respectively. We also observed that adenosine administration had an effect on plasma NO levels in the rats. The plasma NO levels of both the adenosine groups were significantly higher $(P<0.05)$
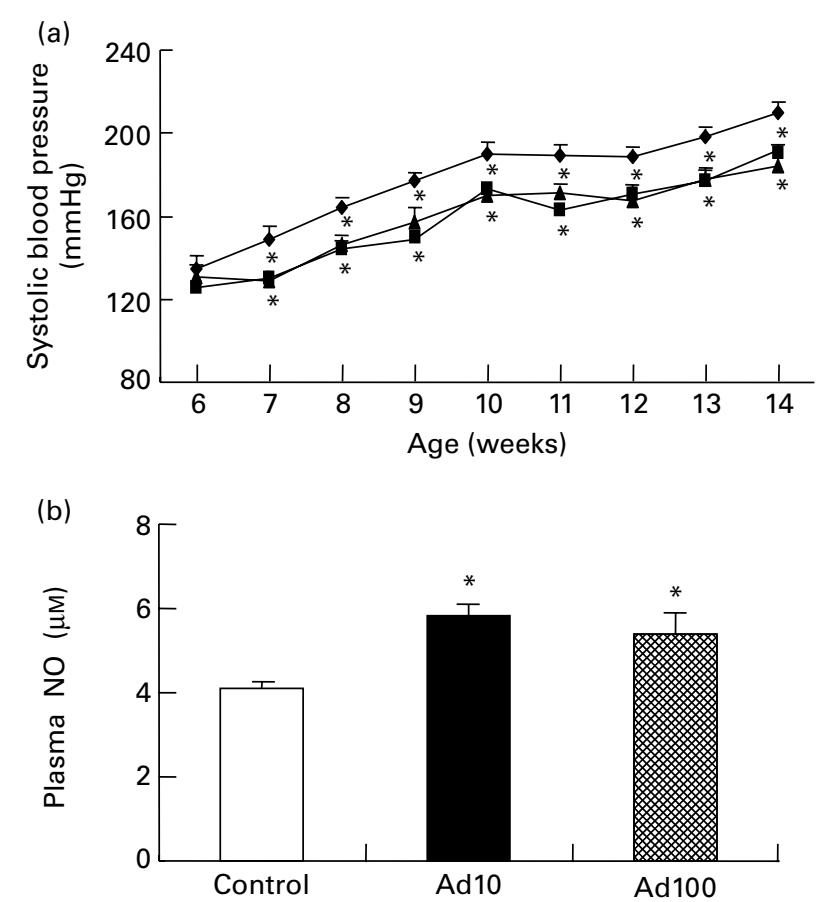

Fig. 1. Effect of adenosine administration on systolic blood pressure (a) and plasma nitric oxide production (b) in rats (mean values with their standard errors of four rats per group). ${ }^{*}$ Mean values were significantly different from control group $(P<0.05)$. $-\$-$, Control; $-\mathbf{-}-$, adenosine $10 \mathrm{mg} / \mathrm{l}$ (Ad10); - $\Delta-$, adenosine $100 \mathrm{mg} / \mathrm{l}$ (Ad100). 
Table 3. Effect of adenosine on plasma total cholesterol, HDL-cholesterol, LDL-cholesterol, TAG, NEFA, blood urea nitrogen (BUN), creatinine, BUN/creatinine and liver total lipid, total cholesterol and TAG levels

(Mean values with their standard errors of four rats per group)

\begin{tabular}{|c|c|c|c|c|c|c|}
\hline \multirow[b]{2}{*}{ Biochemical parameters } & \multicolumn{2}{|c|}{ Control } & \multicolumn{2}{|c|}{ Ad10 } & \multicolumn{2}{|c|}{ Ad100 } \\
\hline & Mean & SEM & Mean & SEM & Mean & SEM \\
\hline \multicolumn{7}{|l|}{ Plasma } \\
\hline Total cholesterol (mg/l) & $795^{a}$ & 39 & $702^{\mathrm{b}}$ & 28 & $798^{a}$ & 38 \\
\hline HDL-cholesterol (mg/l) & $355^{\mathrm{a}}$ & 9 & $431^{\mathrm{b}}$ & 16 & $415^{\mathrm{b}}$ & 18 \\
\hline LDL-cholesterol (mg/l) & $299^{a}$ & 27 & $212^{\mathrm{b}}$ & 30 & $276^{a}$ & 24 \\
\hline TAG $(\mathrm{mg} / \mathrm{l})$ & $704^{a}$ & 50 & $467^{b}$ & 37 & $537^{\mathrm{b}}$ & 62 \\
\hline NEFA (mEq/l) & $0.48^{\mathrm{a}}$ & 0.03 & $0.34^{b}$ & 0.02 & $0.42^{\mathrm{a}}$ & 0.02 \\
\hline BUN (mg/l) & $135^{\mathrm{a}}$ & 4 & $113^{\mathrm{b}}$ & 5 & $114^{\mathrm{b}}$ & 5 \\
\hline Creatinine (mg/l) & $6 \cdot 2^{\mathrm{a}}$ & 0.1 & $5 \cdot 2^{\mathrm{b}}$ & 0.2 & $5 \cdot 7^{\mathrm{b}}$ & 0.2 \\
\hline BUN/creatinine & $21 \cdot 7$ & 0.9 & $21 \cdot 7$ & 0.9 & $20 \cdot 4$ & 1.6 \\
\hline \multicolumn{7}{|l|}{ Liver } \\
\hline Total lipid $(\mathrm{mg} / \mathrm{g})$ liver & $79 \cdot 6$ & 6.5 & $82 \cdot 2$ & $8 \cdot 6$ & $85 \cdot 7$ & 3.4 \\
\hline Total cholesterol $(\mathrm{mg} / \mathrm{g})$ liver & $6 \cdot 1$ & 0.7 & $5 \cdot 8$ & 0.3 & $7 \cdot 3$ & 0.5 \\
\hline TAG $(\mathrm{mg} / \mathrm{g})$ liver & 24.4 & $2 \cdot 1$ & $22 \cdot 0$ & 1.7 & 27.5 & 1.6 \\
\hline
\end{tabular}

Ad10, Adenosine $10 \mathrm{mg} / \mathrm{l}$; Ad100, Adenosine $100 \mathrm{mg} / \mathrm{l}$.

a,b Mean values within a row with unlike superscript letters were significantly different $(P<0.05)$.

than that of the control group (Fig. 1(b)). This result corresponded well with the hypotensive effect of adenosine shown in Fig. 1(a).

\section{Effects of adenosine on plasma parameters}

Table 3 summarises the changes in lipid profile and plasma parameters of kidney function recorded in the present study. After consumption of a high-fat diet, the adenosine groups had significantly lower plasma TAG, blood urea nitrogen and creatinine levels $(P<0.05)$ and increased plasma HDL-cholesterol levels compared with the control group. However, there was no difference in the liver lipid levels between the three groups. Administration of $10 \mathrm{mg} / \mathrm{l}$ adenosine caused significant decreases in the level of TC and LDL-cholesterol compared with the controls and the $100 \mathrm{mg} / \mathrm{l}$ adenosine group. After 8 weeks of adenosine intake, the rats showed significant decreases in plasma glucose and insulin levels $(P<0.05)$ compared with the control animals (Table 4). Analysis of plasma adiponectin levels revealed that both the adenosine groups had significant increases compared with the control group (Fig. 2). Furthermore, adenosine treatment $(10 \mu \mathrm{M})$ resulted in significant increases in adiponectin levels in the culture medium of 3T3-L1 adipocyte cells after $6 \mathrm{~h}$. The adiponectin level was $155 \cdot 5$ (SEM 5.3) and $118.8(\mathrm{SEM} 10 \cdot 5) \mathrm{ng} / \mathrm{ml}$ in the test and the control groups, respectively.
Urinary 8-OHdG has been used widely as a sensitive biomarker of oxidative DNA damage and total systemic oxidative stress. The effect of adenosine on urinary $8-\mathrm{OHdG}$ level is shown in Fig. 3. Both the adenosine groups had significant decreases in urinary 8-OHdG levels compared with the control group.

In the present study, we carried out an oral glucose tolerance test at a dose of $1.8 \mathrm{~g}$ glucose solution $/ \mathrm{kg}$ body weight (Figs. 4(a) and 5(a)) to determine whether adenosine administration improved glucose clearance after intake of a high-fat diet. During the oral glucose tolerance test, blood glucose and insulin levels at 30 and 60 min were decreased in both the adenosine groups compared with the control group. In addition, in the adenosine groups, the incremental areas under the curve of plasma glucose and insulin concentrations were significantly lower than those in the control group (Figs. 4(b) and 5(b)).

\section{Gene expression levels}

We investigated the effect of adenosine administration on mRNA expression levels in the liver and perirenal fat using the quantitative RT-PCR method (Table 5). The mRNA expression level of genes related to anti-oxidative activity such as superoxide dismutase was significantly upregulated in Ad10 group, and tended to be higher in Ad100 group than in the control group. In accordance with our finding that plasma adiponectin levels were increased after adenosine

Table 4. Effect of adenosine on plasma glucose and insulin levels

(Mean values with their standard errors of four rats per group)

\begin{tabular}{|c|c|c|c|c|c|c|}
\hline \multirow[b]{2}{*}{ Biochemical parameters } & \multicolumn{2}{|c|}{ Control } & \multicolumn{2}{|c|}{ Ad10 } & \multicolumn{2}{|c|}{ Ad100 } \\
\hline & Mean & SEM & Mean & SEM & Mean & SEM \\
\hline Glucose (mg/l) & $1686^{a}$ & 55 & $1484^{b}$ & 24 & $1531^{b}$ & 66 \\
\hline Insulin (ng/ml) & $1.8^{\mathrm{a}}$ & 0.2 & $1 \cdot 1^{\mathrm{b}}$ & 0.1 & $1 \cdot 2^{\mathrm{b}}$ & 0.1 \\
\hline
\end{tabular}

Ad10, Adenosine $10 \mathrm{mg} / \mathrm{l}$; Ad100, Adenosine $100 \mathrm{mg} / \mathrm{l}$.

a,b Mean values within a row with unlike superscript letters were significantly different $(P<0 \cdot 05)$. 


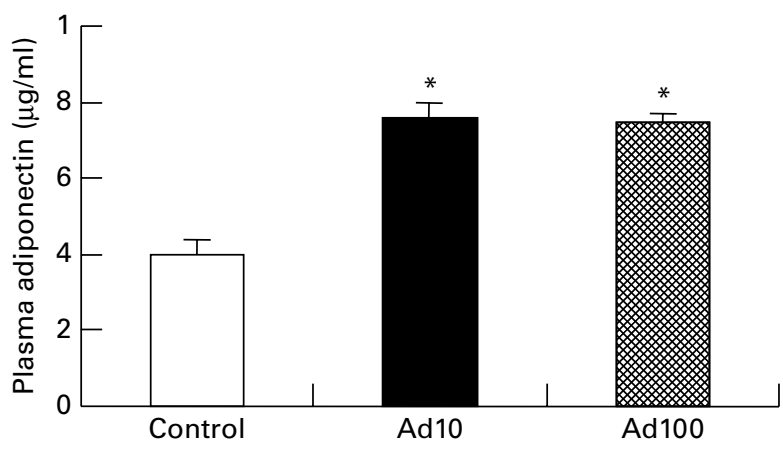

Fig. 2. Effect of adenosine administration on plasma adiponectin in rats (mean values with their standard errors of four rats per group). ${ }^{*}$ Mean values were significantly different from control group $(P<0 \cdot 05)$. Ad10, Adenosine $10 \mathrm{mg} / \mathrm{l}$; Ad100, Adenosine $100 \mathrm{mg}$.

administration (Fig. 2), we confirmed that mRNA expression of adiponectin and adiponectin receptor 1 in perirenal fat was also upregulated compared with the control. In addition, we found that the mRNA expression level of the adiponectin receptor 2 was higher in the adenosine groups than in the control group. Furthermore, we determined the mRNA expression level of adenosine receptors in the liver, and found that the adenosine receptor $\mathrm{A}_{2 \mathrm{~B}}$ was significantly downregulated in the Ad10 group and tended to be lower in the Ad100 group than in the control group. There was, however, no difference in the mRNA expression level of the adenosine receptors $A_{2 a}$ and $A_{3}$ between the three groups.

\section{Discussion}

We demonstrated, for the first time to our knowledge, that oral administration of low (Ad10)- and high (Ad100)- dose adenosine in SHRSP fed a high-fat diet improved hypertension after 1 week and hyperlipidaemia and hyperglycaemia after 8 weeks of administration. Our results were consistent with those of previous reports ${ }^{(7,8)}$, and also support the findings of our previous study in SHRSP fed a normal diet ${ }^{(11)}$. We chose to study SHRSP fed a high-fat diet consisting of lard as these rats exhibit a spontaneous hypertension, hyperlipidaemia and insulin resistance ${ }^{(20,21)}$. The concentration of adenosine in cells and tissue fluids was estimated between 10 and $100 \mathrm{~nm}$. Adenosine is mainly formed by the breakdown

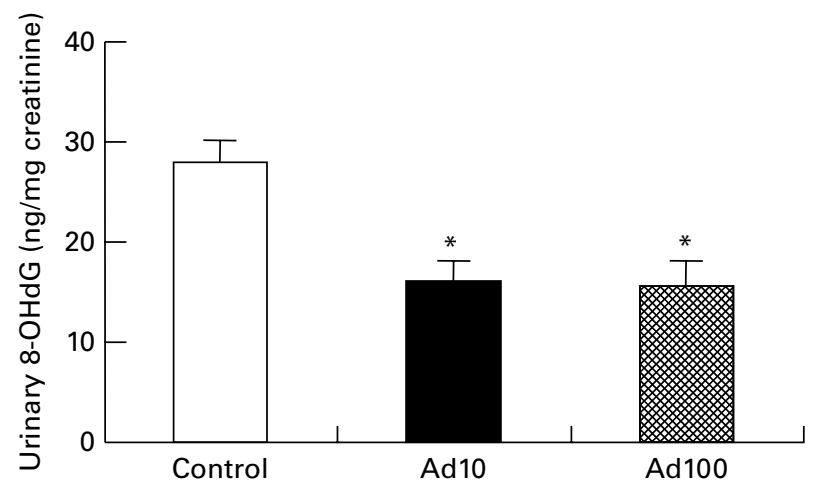

Fig. 3. Effect of adenosine administration on urinary 8-hydroxy-2'-deoxyguanosine $(8-\mathrm{OHdG})$ in rats (mean values with their standard errors of four rats per group). ${ }^{*}$ Mean values were significantly different from control group $(P<0.05)$. Ad10, Adenosine $10 \mathrm{mg} / \mathrm{l} ;$ Ad100, Adenosine $100 \mathrm{mg}$.
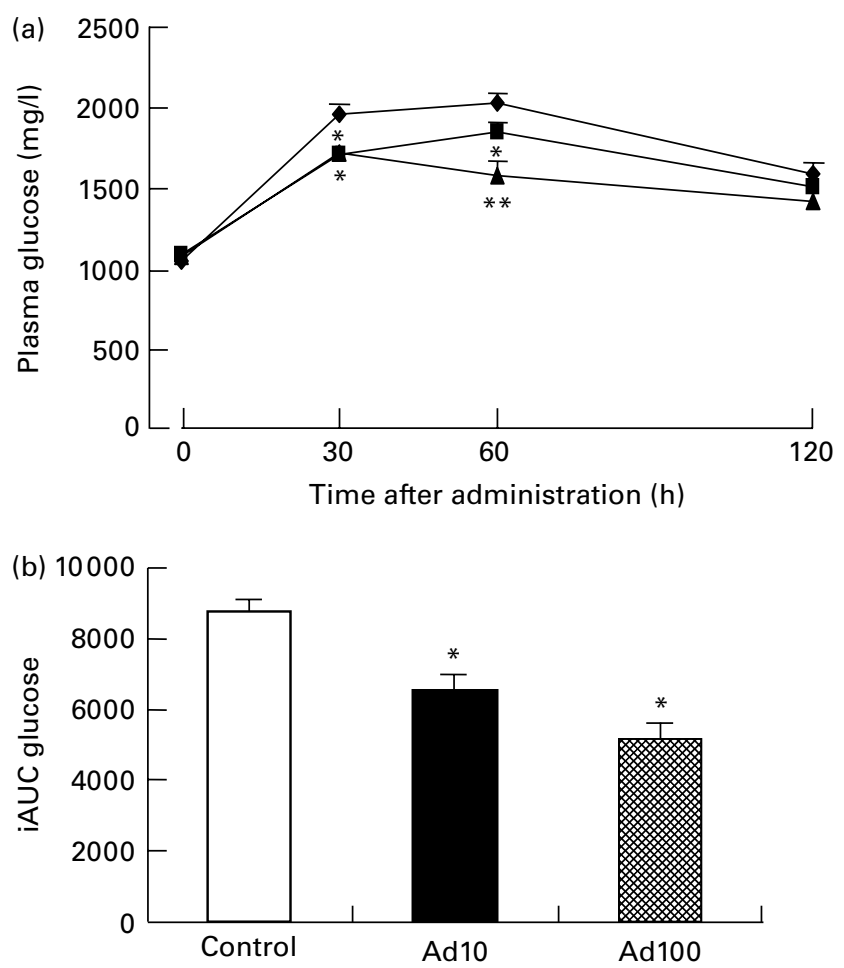

Fig. 4. Changes in plasma glucose (a) and incremental area under the curve (iAUC) (b) determined by the oral glucose tolerance test (OGTT) in rats (mean values with their standard errors of four rats per group). Mean values were significantly different from those of the control group: ${ }^{\star} P<0.05,{ }^{\star} P=0.01$.

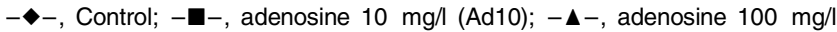
(Ad100).

of intracellular or extracellular adenine nucleotides ${ }^{(6)}$. Two main enzymes, adenosine deaminase and adenosine kinase, play a key role in the catabolisation of adenosine. When adenosine enters the blood circulation, it is cleaved by adenosine deaminase, which is present in blood vessel wall ${ }^{(22)}$. Future study is needed to explain the underlying bioavailability of adenosine in the present study.

It is well established that NO produced by endothelial cells plays a pivotal role in the maintenance of vascular function and health ${ }^{(23)}$ by elevating intracellular cyclic GMP levels, resulting in smooth muscle relaxation ${ }^{(24)}$. Several studies have also reported that impaired NO release from endothelial cells $^{(25)}$ is related to the increase in BP that occurs in SHRSP. In the present study, we observed that administration of low- or high-dose adenosine resulted in increased plasma NO levels (Fig. 1(b)), and that this change corresponded closely with the hypotensive effect in SHRSP (Fig. 1(a)). We consider that the enhanced plasma NO level observed was a consequence of the vasodilatory effect of adenosine administration, and that the hypotensive effect of adenosine may be due to the potent vasodilation induced by adenosine following the activation of adenosine receptors $\left(\mathrm{A}_{2}\right)$ in vascular smooth muscle ${ }^{(26)}$. We therefore propose that the mechanism underlying the hypotensive effect of adenosine is based on increased plasma NO levels, with this increase enhancing NO-mediated vasodilatory tone and accounting for the observed amelioration of hypertension. We consider that low- and high-dose adenosine administration may therefore improve depressed vasodilation in SHRSP. 

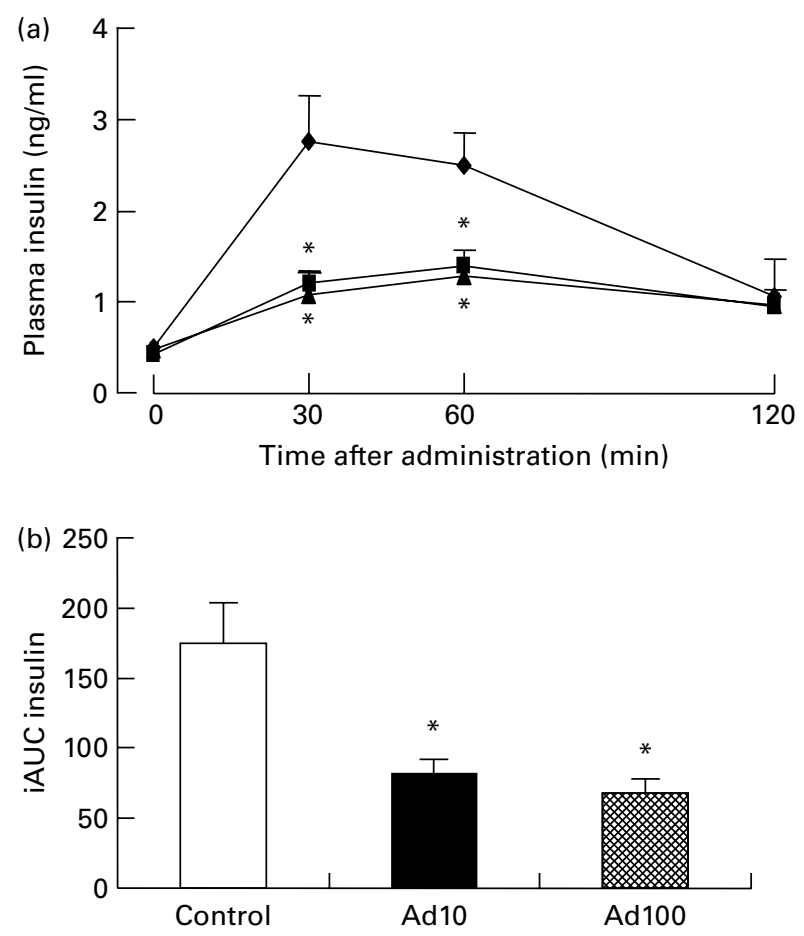

Fig. 5. Changes in plasma insulin (a) and incremental area under the curve (iAUC) (b) determined by the oral glucose tolerance test (OGTT) in rats (mean values with their standard errors of four rats per group). * Mean values were significantly different from control group $(P<0.01)$. $-\bullet-$, Control; -ם-, Ad10; - $\Delta-$ Ad100.

We also investigated the hepatic mRNA expression level of adenosine receptors in order to elucidate the mechanism by which adenosine administration improved lipid metabolism. In line with previous studies ${ }^{(27-30)}$, we confirmed that adenosine $A_{1}, A_{2 A}, A_{2 B}$ and $A_{3}$ receptors are clearly expressed on

Table 5. Effect of adenosine on liver and perirenal fat mRNA expression levels expressed as relative increases or decreases as determined by quantitative RT-PCR

(Mean values with their standard errors of six rats per group)

\begin{tabular}{|c|c|c|c|c|c|c|}
\hline \multirow{2}{*}{ Gene } & \multicolumn{2}{|c|}{ Control } & \multicolumn{2}{|c|}{ Ad10 } & \multicolumn{2}{|c|}{ Ad100 } \\
\hline & Mean & SEM & Mean & SEM & Mean & SEM \\
\hline \multicolumn{7}{|l|}{ Liver } \\
\hline Sod & $1 \cdot 0^{\mathrm{a}}$ & 0.2 & $1 \cdot 8^{\mathrm{b}}$ & 0.3 & $1 \cdot 6^{a, b}$ & 0.4 \\
\hline Gshpx & 1.0 & 0.2 & 1.0 & 0.3 & 0.6 & 0.1 \\
\hline Adipor2 & $1 \cdot 0$ & 0.2 & 2.5 & 0.7 & 2.5 & $1 \cdot 2$ \\
\hline Adora2a & 1.0 & 0.1 & 1.0 & 0.1 & $1 \cdot 2$ & 0.3 \\
\hline Adora2b & $1 \cdot 0^{a}$ & 0.2 & $0.6^{\mathrm{b}}$ & 0.1 & $0.7^{a, b}$ & 0.1 \\
\hline Adora3 & $1 \cdot 0$ & 0.2 & 0.8 & 0.1 & $1 \cdot 0$ & 0.2 \\
\hline \multicolumn{7}{|l|}{ Perirenal fat } \\
\hline Ppary & $1 \cdot 0$ & 0.3 & 1.4 & 0.4 & 1.7 & 0.7 \\
\hline Srebp1c & 1.0 & 0.2 & 1.0 & 0.2 & 1.0 & 0.3 \\
\hline Fasn & 1.0 & 0.2 & $1 \cdot 2$ & 0.5 & 1.4 & 0.3 \\
\hline Adipoq & 1.0 & 0.2 & $2 \cdot 2$ & 0.7 & 2.5 & 0.7 \\
\hline Adipor1 & $1 \cdot 0^{\mathrm{a}}$ & 0.3 & $1 \cdot 6^{a, b}$ & 0.4 & $3 \cdot 3^{\mathrm{b}}$ & $1 \cdot 2$ \\
\hline
\end{tabular}

Ad10, Adenosine $10 \mathrm{mg} / \mathrm{l}$; Ad100, Adenosine $100 \mathrm{mg} /$; Sod, superoxide dismutase; Gshpx, glutathione peroxidase; Adipor2, adiponectin receptor 2; Adora2a, adenosine $A_{2 A}$ receptor; Adora2b, adenosine $A_{2 B}$ receptor; Adora3, adenosine $A_{3}$ receptor; Ppar $\gamma$, PPAR $\gamma$; Srebp1c, sterol regulatory element-binding protein-1c; Fasn, fatty acid synthase; Adipoq, Adiponectin; Adipor1, adiponectin receptor 1.

a,b Mean values within a row with unlike superscript letters were significantly different $(P<0.05)$. hepatocytes, and showed that the hepatic mRNA expression levels of adenosine $A_{2 A}, A_{2 B}$ and $A_{3}$ receptors were altered by adenosine administration (Table 5). Previous studies indirectly provide evidence to support the role of adenosine and its receptors in the pathogenesis of fatty liver ${ }^{(31,32)}$. In the present study, we found that Ad10 significantly downregulated and Ad100 tended to downregulate the mRNA expression level of the adenosine $A_{2 B}$ receptor. $A$ recent study showed that the adenosine $\mathrm{A}_{2 \mathrm{~B}}$ receptor regulates the phosphorylation of a critical signalling molecule that controls the pathways of hepatic fatty acid oxidation ${ }^{(33)}$. From this viewpoint, our results suggest that regulation of lipid metabolism associated with the administration of low-dose adenosine (Ad10) is mediated by adenosine receptors. Our observations also suggest that lipid metabolism in SHRSP may be regulated by a different mechanism compared with the animals administered adenosine in drinking water, although further study is required to clarify this.

Our data show that urinary $8-\mathrm{OHdG}$ concentration was higher in the control group than in the adenosine groups, suggesting that increased oxidative stress may have a primary pathogenic role in SHRSP (Fig. 3). It has been reported that $8-\mathrm{OHdG}$ level is increased significantly in obese diabetic KKAy mice in comparison with C57BL mice ${ }^{(34)}$, and that 8-OHdG occurs in multiple tissues of streptozotocin-induced diabetic rats ${ }^{(35)}$. We also observed that mRNA expression level of genes related to anti-oxidative activity such as superoxide dismutase was upregulated significantly by adenosine administration (Table 5). Our results imply that adenosine ameliorates oxidative capacity-related factors along with improving diabetic condition in SHRSP. On the other hand, it has been demonstrated that high-fat diet decreases insulin sensitivity ${ }^{(36)}$. As seen in Table 4 , adenosine administration may improve insulin sensitivity despite consumption of a high-fat diet. The results of the oral glucose tolerance test clearly showed that adenosine administration suppressed the rapid increase in plasma glucose and insulin levels and the incremental areas under the curve (Figs. 4 and 5). Taken together, these results suggest that adenosine contributes to improving the regulation of glucose metabolism in SHRSP fed a high-fat diet.

It is well known that adiponectin plays a central role in antagonising the metabolic parameters involved in obesity, hepatic lipid deposition and inflammation that ultimately lead to systemic insulin resistance ${ }^{(37)}$. Our previous results suggest that an enhanced plasma adiponectin level alleviates hyperinsulinaemia, and may prevent the development of hypertension in SHRSP fed a normal $\operatorname{diet}^{(11)}$. In the present study, we also found that adenosine administration increased plasma adiponectin levels (Fig. 2) and improved hyperinsulinaemia in rats fed a high-fat diet (Table 4). Furthermore, increased adiponectin expression may have an impact on lipid levels in obese mice, as it has been reported that HDL-cholesterol levels are increased by adiponectin in genetic mouse models of obesity $(o b / o b \text { and } d b / d b)^{(38-40)}$. In the present study, low- and high-dose adenosine administration resulted in significantly lower total plasma TC and increased HDL-cholesterol levels compared with the controls (Table 3). We therefore consider that enhanced plasma adiponectin level resulting from daily intake of adenosine may be caused by the upregulation of the mRNA levels of 
adiponectin and its receptors in perirenal fat and liver (Table 5). As adiponectin and adiponectin receptors represent potential versatile therapeutic targets to combat obesityrelated diseases characterised by insulin resistance ${ }^{(41)}$ and to ameliorate hyperglycaemia and hyperinsulinaemia thereby protecting against diabetes ${ }^{(42)}$, we suggest that adenosine is effective for improving metabolic syndrome-related parameters in SHRSP.

In conclusion, the present results are what we consider to be novel findings on the effects of low- and high-dose adenosine administration in SHRSP fed a high-fat diet. Our results clearly indicate that dietary adenosine is effective in increasing insulin sensitivity and plasma adiponectin levels and also in upregulating mRNA levels of adiponectin and its receptors in perirenal fat and liver and in downregulating hepatic mRNA levels of adenosine $\mathrm{A}_{2 \mathrm{~B}}$ receptor. We propose that these effects mediate the effect of adenosine on metabolic activity including the lowering of BP and improvement in glucose and lipid metabolism. The mechanism by which the oral administration of adenosine mediates this process, however, requires further investigation. In this regard, we plan to perform clinical studies to confirm the anti-metabolic syndrome activity of adenosine in human subjects, in addition to carrying out further experiments in animal models.

\section{Acknowledgements}

This research was partially supported by a Grant-in-Aid for Scientific Research from the Japan Society for the Promotion of Science to A. and H. S. (no. 19.07 166), and a Grant for the City Area Program from the Ministry of Education, Culture, Sports, Science and Technology of Japan to T. K. There are no conflicts of interest in relation to the present paper. All authors are responsible for the content of the manuscript. A. A. carried out majority of the analytical work, designed the experiment and wrote the manuscript. Y. S. contributed to the in vitro experimental work. T. K. was involved in the experiments and discussion of the experimental results. H. S. and $\mathrm{M}$. K. contributed to the supervision and drafting of the manuscript and to the design of some of the experiments.

\section{References}

1. Haffner S \& Taegtmeyer H (2003) Epidemic obesity and the metabolic syndrome. Circulation 108, 1541-1545.

2. Sharma AM (2006) The obese patient with diabetes mellitus: from research targets to treatment options. Am J Med 119, 17-23.

3. Hardus PM, van Vuuren CL, Crawford D, et al. (2003) Public health perceptions of the causes and prevention of obesity among primary school children. Int J Obes Relat Metab Disord 27, 1465-1471.

4. Jackson EK \& Dubey RK (2001) Role of the extracellular cAMP-adenosine pathway in renal physiology. Am J Physiol 28, F597-F612.

5. Olah SN \& Stiles GL (2000) The role receptor structure in determining adenosine receptor activity. Pharmacol Ther 85, 55-75.

6. Zimmermann H (2000) Extracellular metabolism of ATP and other nucleotides. Naunyn Schmiedebergs Arch Pharmacol 362, 299-309.

7. Fukumori Y, Maeda N, Takeda H, et al. (2000) Serum glucose and insulin response in rats administered with sucrose or starch containing adenosine, inosine or cytosine. Biosci Biotechnol Biochem 64, 237-243.

8. Fukumori Y, Takeda H, Fujisawa T, et al. (2000) Blood glucose and insulin concentrations are reduced in human administered sucrose with inosine or adenosine. J Nutr 130, 1946-1949.

9. Motti A, Tissot C, Rimensberger PC, et al. (2006) Intravenous adenosine for refractory pulmonary hypertension in low-weight premature newborn: a potential new drug for rescue therapy. Pediatr Crit Care Med 7, 380-382.

10. Dubey RK, Gillespie DG, Mi Z, et al. (2005) Adenosine inhibit PDGF-induced growth of human glomerular mesangial cells via $\mathrm{A}_{2 \mathrm{~B}}$ receptors. Hypertension 46, 628-634.

11. Ardiansyah, Shirakawa H, Shimeno T, et al. (2009) Adenosine, an identified active component from the Driselase-treated fraction of rice bran, is effective at improving metabolic syndrome in stroke-prone spontaneously hypertensive rats. J Agric Food Chem 57, 2558-2564.

12. Chan ES, Montesinos MC, Fernandez P, et al. (2006) Adenosine $\mathrm{A}(2 \mathrm{~A})$ receptors play a role in the pathogenesis of hepatic cirrhosis. Br J Pharmacol 148, 1144-1155.

13. Peng Z, Fernandez P, Wilder T, et al. (2008) Ecto-5ı-nucleotidase (CD73)-mediated extracellular adenosine production plays a critical role in hepatic fibrosis. FASEB J 22, 2263-2272.

14. Dhalla AK, Wong MY, Voshol PJ, et al. (2007) A1 adenosine receptor partial agonist lowers plasma FFA and improves insulin resistance induced by high-fat diet in rodents. Am $J$ Physiol Endocrinol Metab 292, E1358-E1363.

15. Ardiansyah, Shirakawa H, Koseki T, et al. (2006) Rice bran fractions improve blood pressure, lipid profile, and glucose metabolism in stroke-prone spontaneously hypertensive rats. J Agric Food Chem 54, 1914-1920.

16. Friedewald WT, Levy RI \& Fredrickson DS (1972) Estimation of the concentration of low-density lipoprotein cholesterol in plasma without use of the preparative ultracentrifuge. Clin Chem 18, 499-502.

17. Folch J, Lees M \& Stanley GHS (1957) A simple method for the isolation and purification of total lipid from animal tissues. J Biol Chem 226, 497-509.

18. Wolever TM \& Jenkins DJ (1986) The use of the glycemic index in predicting the blood glucose response to mixed meals. Am J Clin Nutr 43, 167-172.

19. Shirakawa H, Ohsaki Y, Minegishi Y, et al. (2006) Vitamin K deficiency reduces testosterone production in the testis through down-regulation of the Cyp11a a cholesterol side chain cleavage enzyme in rats. Biochim Biophys Acta 1760, $1482-1488$.

20. Aitman TJ, Gotoda T, Evans AL, et al. (1997) Quantitative trait loci for cellular defects in glucose and fatty acid metabolism in hypertensive rats. Nat Genet 16, 197-201.

21. Collison M, Glazier AM, Graham D, et al. (2000) Cd36 and molecular mechanisms of insulin resistance in the strokeprone spontaneously hypertensive rat. Diabetes 49, 2222-2226.

22. Hershfield MS (2005) New insights into adenosine-receptormediated immunosuppression and the role of adenosine in causing the immunodeficiency associated with adenosine deaminase deficiency. Eur J Immunol 35, 25-30.

23. Kibbe M, Billiar T \& Tzeng E (1999) Inducible nitric oxide synthase and vascular injury. Cardiovasc Res 43, 650-657.

24. Moncada S, Higgs E \& Palmer RMJ (1991) Nitric oxide: Physiology, pathophysiology and pharmacology. Pharmacol Rev 43, 109-142.

25. Malinski T, Kapturczak M, Dayharsh J, et al. (1993) Nitric oxide synthase activity in genetic hypertension. Biochem Biophys Res Commun 194, 654-658.

26. Fullerton DA, Agrafojo J \& McIntyre RC Jr (1996) Pulmonory vascular smooth muscle relaxation by cAMP-mediated pathways. J Surg Res 61, 444-448. 
27. Guinzberg R, Laguna I, Zentella A, et al. (1987) Effect of adenosine and inosine on ureagenesis in hepatocytes. Biochem $J$ 245, 371-374.

28. Guinzberg R, Diaz-Cruz A, Uribe S, et al. (1993) Inhibition of adenosine mediated responses in isolated hepatocytes by depolarizing concentrations of $\mathrm{K}^{+}$. Biochem Biophys Res Commun 197, 229-234.

29. Tinton SA, Lefebvre VH, Cousin OC, et al. (1993) Cytolytic effects and biochemical changes induced by extracellular ATP to isolated hepatocytes. Biochim Biophys Acta 1176, 1-6.

30. Gonzalez-Benitez E, Guinzberg R, Diaz-Cruz A, et al. (2002) Regulation of glycogen metabolism in hepatocytes through adenosine receptors: role of $\mathrm{Ca}^{2+}$ and cAMP. Eur $J$ Pharmacol 437, 105-111.

31. Muroyama K, Murosaki S, Yamamoto Y, et al. (2003) Anti-obesity effects of a mixture of thiamin, arginine, caffeine, and citric acid in non-insulin dependent diabetic KK mice. J Nutr Sci Vitaminol 49, 56-63.

32. Murosaki S, Lee TL, Muroyama K, et al. (2007) A combination of caffeine, arginine, soy isoflavones, and L-carnitine enhances both lipolysis and fatty acid oxidation in 3T3-L1 and HepG2 cells in vitro and in KK mice in vivo. J Nutr 137, 2252-2257.

33. Peng Z, Borea PA, Varani K, et al. (2009) Adenosine signaling contributes to ethanol-induces fatty liver in mice. J Clin Invest 119, $582-594$.

34. Kanaya Y, Doi T, Sasaki H, et al. (2004) Rice bran extract prevent the elevation of plasma peroxylipid in KKAy diabetic mice. Diabetes Metab Res Rev 66S, S157-S160.
35. Hsieh RH, Lien LM, Lin SH, et al. (2005) Alleviation of oxidative damage in multiple tissues in rats with streptozotocin-induced diabetes by rice bran oil supplementation. Ann N Y Acad Sci 1042, 365-371.

36. Friedman JM (2000) Obesity in the new millennium. Nature 404, 632-634.

37. Kim JY, van de Wall E, Laplante M, et al. (2007) Obesityassociated improvements in metabolic profile through expansion of adipose tissue. J Clin Invest 117, 2621-2637.

38. Silver DL, Jiang XC \& Tall AR (1999) Increased high density lipoprotein (HDL), defective hepatic catabolism of ApoA-I and ApoA-II, and decreased ApoA-I mRNA in ob/ob mice: possible role of leptin in stimulation of HDL turnover. $J$ Biol Chem 274, 4140-4146.

39. Lundasen T, Liao W, Angelin B, et al. (2003) Leptin induces the hepatic high density lipoprotein receptor scavenger receptor B type I (SR-BI) but not cholesterol 7alpha-hydroxylase (Cyp7a1) in leptin-deficient (ob/ob) mice. J Biol Chem 278, 43224-43228.

40. Gruen ML, Plummer MR, Zhang W, et al. (2005) Persistence of high density lipoprotein particles in obese mice lacking apolipoprotein A-I. J Lipid Res 46, 2007-2014.

41. Kadowaki T, Yamauchi T, Kubota N, et al. (2006) Adiponectin and adiponectin receptors in insulin resistance, diabetes, and the metabolic syndrome. J Clin Invest 116, 1784-1792.

42. Yamauchi T, Kamon J, Waki H, et al. (2001) The fat-derived hormone adiponectin reverse insulin resistance associated with both lipoatrophy and obesity. Nat Med 7, 941-946. 